\title{
Daytime restricted feeding modifies the daily regulation of fatty acid $\beta$-oxidation and the lipoprotein profile in rats
}

\author{
J. B. Rivera-Zavala ${ }^{1}$, C. Molina-Aguilar ${ }^{1}$, M. Pérez-Mendoza ${ }^{2}$, M. Olguín-Martínez ${ }^{3}$, R. Hernández-Muñoz ${ }^{3}$, \\ G. A. Báez-Ruiz ${ }^{4}$ and M. Díaz-Muñoz ${ }^{1 *}$ \\ ${ }^{1}$ Departamento de Neurobiología Celular y Molecular, Instituto de Neurobiología, Universidad Nacional Autónoma de México, \\ Campus UNAM-Juriquilla, Querétaro, Mexico 76230 \\ ${ }^{2}$ Facultad de Ciencias, Unidad Multidisciplinaria de Docencia e Investigación, Universidad Nacional Autónoma de México, \\ Campus UNAM-Juriquilla, Querétaro, Mexico 76230 \\ ${ }^{3}$ Departamento de Biología Celular y Desarrollo, Instituto de Fisiología Celular, Ciudad Universitaria, Universidad Nacional \\ Autónoma de México, Ciudad de México, Mexico 04510 \\ ${ }^{4}$ Facultad de Ciencias, Universidad Autónoma de San Luis Potosí, San Luis Potosí, Mexico 78000 \\ (Submitted 14 December 2016 - Final revision received 20 February 2017 - Accepted 9 March 2017 - First published online 9 May 2017)
}

\section{Abstract}

Daytime restricted feeding ( $2 \mathrm{~h}$ of food access from 12.00 to 14.00 hours for 3 weeks) is an experimental protocol that modifies the relationship between metabolic networks and the circadian molecular clock. The precise anatomical locus that controls the biochemical and physiological adaptations to optimise nutrient use is unknown. We explored the changes in liver oxidative lipid handling, such as $\beta$-oxidation and its regulation, as well as adaptations in the lipoprotein profile. It was found that daytime restricted feeding promoted an elevation of circulating ketone bodies before mealtime, an altered hepatic daily rhythmicity of ${ }^{14} \mathrm{CO}_{2}$ production from radioactive palmitic acid, and an up-regulation of the fatty acid oxidation activators, the $\alpha$-subunit of AMP-activated protein kinase (AMPK), the deacetylase silent mating type information regulation homolog 1 , and the transcriptional factor PPAR $\gamma-1 \alpha$ coactivator. An increased localisation of phosphorylated $\alpha$-subunit of AMPK in the periportal hepatocytes was also observed. Liver hepatic lipase $\mathrm{C}$, important for lipoprotein transformation, showed a change of daily phase with a peak at the time of food access. In serum, there was an increase of LDL, which was responsible for a net elevation of circulating cholesterol. We conclude that our results indicate an enhanced fasting response in the liver during daily synchronisation to food access, which involves altered metabolic and cellular control of fatty acid oxidation as well a significant elevation of serum LDL. These adaptations could be part of the metabolic input that underlies the expression of the food-entrained oscillator.

Key words: Food synchronisation: $\beta$-Oxidation: AMP-activated protein kinase: PPAR $\boldsymbol{\gamma}$-1 $\alpha$ coactivator: Liver zonation

Restricted access to food is a circadian synchroniser that modifies liver metabolism ${ }^{(1-3)}$. When food availability is limited (30\% less energy content) to $2 \mathrm{~h}$ during the daytime restricted feeding (DRF) protocol for a prolonged period of time, a change occurs in the coordination between the suprachiasmatic nucleus (SCN) and the rhythmicities of peripheral circadian oscillators $^{(4)}$. This new state in the circadian timing system allows the expression of an alternative circadian clock known as the food-entrained oscillator $(\mathrm{FEO})^{(4,5)}$. The activity of the FEO involves a dynamic mutual interaction between the circadian molecular clock and the metabolic energy networks within a variety of tissues and organs ${ }^{(2-4,6)}$.
Using the DRF protocol, we have reported that some parameters in the handling of lipids by the liver and adipose tissue are modified before food access: (1) peaks of serum NEFA and ketone bodies (KB) are observed, suggesting increased lipolytic activity and fatty acid oxidation (FAO) in the liver ${ }^{(7-9)} ;(2)$ the arrival of NEFA to the liver is associated with activation of PPAR $\alpha^{(9)}$; (3) TAG within the liver are reduced before food access ${ }^{(10)}$; (4) there is an increase in hepatic mitochondrial proton-motive potential, an oxidised redox state and elevated levels of $\mathrm{ATP}^{(11)}$; (5) 2-h food pulses mainly affect lipid metabolites with discreet action on blood levels of glucose and proteins ${ }^{(8)}$; and (6) liver TAG and NEFA showed persistent

Abbreviations: AL, ad libitum; AMPK, AMP-activated protein kinase; BMAL1, brain and muscle Arnt-like protein 1; CPT-1 $\alpha$, carnitine palmitoyltransferase $1 \alpha$; CRY, cryptochrome gene; DRF, daytime restricted feeding; Fa, $21 \mathrm{~h}$ fasting; FAB, food anticipatory behaviour; FAO, fatty acid oxidation; FEO, food-entrained oscillator; KB, ketone bodies; LIPC, hepatic lipase; $\mathrm{pAMPK} \alpha$, phosphorylated AMP-activated protein kinase $\alpha$; PEPCK, phosphoenolpyruvate carboxykinase; PER, period gene; PGC- $1 \alpha$, PPAR $\gamma$ - $1 \alpha$ coactivator; PP, periportal; PV, perivenous; Re, refed after a 21-h fast; SIRT1, silent mating type information regulation homolog 1 . 
oscillations under fasting conditions, suggesting that daily fluctuations of lipid metabolites are driven by a circadian $\operatorname{clock}^{(8)}$

The liver metabolic activity is compartmentalised following an oxygenation gradient, high $\mathrm{O}_{2}$ levels in the periportal (PP) zone, and lower $\mathrm{O}_{2}$ levels in the perivenous (PV) zone. Hepatic lipid metabolism is zonated: lipogenesis occurs mainly in PV hepatocytes, whereas fatty acid-degradation takes place in PP hepatocytes ${ }^{(12)}$. The steps involved in hepatic lipid handling are strictly regulated by the fasting-feeding cycle and the energetic status of the liver ${ }^{(13)}$. As the DRF protocol involves a period of $22 \mathrm{~h}$ without food access, we have explored this fasting response by characterising the biochemical, hormonal and transcriptional regulation of gluconeogenesis ${ }^{(14)}$ as well as the daily variations of ureagenesis ${ }^{(15)}$. In this context, it has been reported that the $24-\mathrm{h}$ profiles of the next metabolic regulatory factors, the silent mating type information regulation homolog 1 (SIRT1) and the PPAR $\gamma-1 \alpha$ coactivator (PGC- $1 \alpha$ ), are modified in response to the DRF protocol. SIRT1 showed higher levels before (11.00 hours) and after food intake (14.00 hours), whereas PGC- $1 \alpha$ showed an increased level during the entire 24-h cycle ${ }^{(14)}$.

Some of the most important parameters in the molecular machinery that coordinate the function of the circadian molecular clock and the cellular energy status are the NAD ${ }^{+}$: $\mathrm{NADH}$ and AMP:ATP ratios. For example, the AMP-activated protein kinase (AMPK) and SIRT1 enzymes are activated by the increase of AMP:ATP and $\mathrm{NAD}^{+}: \mathrm{NADH}$ ratios, respectively ${ }^{(16-18)}$. During fasting response in the liver; AMPK, SIRT1 and PGC- $1 \alpha$ act through the nuclear receptor $\operatorname{PPAR} \alpha^{(19,20)}$. $\operatorname{PPAR} \alpha$ regulates the transcription of key gluconeogenic genes (glucose 6 phosphatase and phosphoenolpyruvate carboxykinase $(P E P C K)$ ), and the expression of the FAO limitingenzyme carnitine palmitoyltransferase $1 \alpha(C P T-1 \alpha)^{(21)}$. CPT-1 $\alpha$ is inhibited by malonyl-CoA in the postprandial state ${ }^{(22)}$.

During the postprandial state, the complex mobilisation of TAG and cholesterol in the liver and among a variety of tissues is carried out by a family of macromolecules known as lipoproteins (LP). LP are classified according to their proportion of TAG and cholesterol, and the nature of their protein moiety: HDL, LDL and VLDL ${ }^{(23)}$. The hepatic TAG lipase (LIPC) is a lipolytic enzyme that contributes to the regulation of plasma TAG levels and the formation of $\mathrm{LDL}^{(24)}$.

It is hypothesised, on the basis of these antecedents, that the DRF protocol in the liver involves the appearance of an emergent relationship between the circadian timing system and the energy metabolic pathways. In this context, the present project is aimed at strengthening the notion that lipid metabolism, especially the part related to the fasting response, will be one of the important adaptive processes in the expression of the FEO.

Specifically, we characterised the 24-h profiles of: (1) the production of ${ }^{14} \mathrm{CO}_{2}$ from ${ }^{14} \mathrm{C}$-palmitic acid; (2) the differential presence in cytosol and nucleus of the following metabolic effectors: AMPK $\alpha$, phosphorylated AMP-activated protein kinase $\alpha$ (pAMPK $\alpha)$, SIRT1 and PGC- $1 \alpha$; (3) hepatic zonation of pAMPK $\alpha$ in PP and PV hepatocytes; and (4) the concentrations of circulating $\mathrm{KB}$, cholesterol, TAG and lipoproteins.

\section{Methods}

\section{Animals and housing}

To ensure homogeneity in the different experimental groups, male albino Wistar rats were obtained exclusively from Instituto de Neurobiología, Campus UNAM-Juriquilla in Querétaro, México. Circadian rhythm protocols involve cycles of $24 \mathrm{~h}$; for our experiments, sampling done in $3 \mathrm{~h}$ intervals allowed us to obtain well-defined daily behavioural and experimental data. In agreement with other $24 \mathrm{~h}$ rhythmic protocols, our experiments were conducted using four animals (control and experimental) in each sampling time ${ }^{(25,26)}$

All experimental procedures followed the criteria described in the guidelines. In addition, they were approved and conducted according to the institutional guide for the care and use of animals under biomedical experimentation (Universidad Nacional Autónoma de México), and conformed to previously recommended international ethical standards ${ }^{(27)}$. The experiments were performed in accordance with the Mexican Laws for Animal Care (Norma Oficial Mexicana SAGARPA; Secretaría de Agricultura, Ganadería, Desarrollo Rural, Pesca y Alimentación) and the relevant rules set forth by the Mexican Ministry of Health. Adult male Wistar rats, weighing 180 (SEM 20) g at the beginning of the experiment, were maintained in a $12 \mathrm{~h}$ light $-12 \mathrm{~h}$ dark cycle (lights on at 08.00 hours $)$ at constant temperature $\left(22 \pm 1^{\circ} \mathrm{C}\right)$. The light intensity at the surface of the cages averaged 350 luxes. Animals were kept in groups of four in transparent acrylic cages $(40 \times 50 \times 20 \mathrm{~cm})$ with free access to water and food unless stated otherwise.

\section{Experimental design}

Control and experimental groups were as previously reported ${ }^{(14)}$. In brief, rats were randomly assigned to one of the following feeding conditions for 3 weeks: (1) the control rats had ad libitum (AL), free access to food and water throughout the 24-h period; and (2) in the DRF rats food access was limited to 2-h daily, from 12.00 to 14.00 hours. At the end of the feeding protocol, different subgroups of animals were beheaded with a guillotine-like device at 3-h intervals over a 24-h period, starting at 08.00 hours. In addition, two more acute feeding control groups were included. (3) In the first group, animals were fed AL for 3 weeks; on the last day, food was removed at 14.00 hours, and the rats were killed at 11.00 hours the next day (after a $21 \mathrm{~h}$ fasting $(\mathrm{Fa})$ period); (4) the second group of rats was similarly acutely deprived of food for $21 \mathrm{~h}$, then refed for $2 \mathrm{~h}$ (Re) (from 12.00 to 14.00 hours), and killed at 14.00 hours. The experiments were performed following a transverse design to satisfy chronobiological standards. Our protocol used four animals chosen randomly at each sampling time; hence, the total number of experimental animals was sixtyfour, including AL-fed and DRF groups. In addition, eight more rats were considered in our protocol for control of feeding condition (acute Fa and Re groups).

\section{Blood and liver sampling}

Blood samples were collected in vacutainer tubes and then centrifuged at $5000 \mathrm{rpm}$ for $5 \mathrm{~min}$ to obtain serum. The liver was 
processed for subcellular fractionation (nucleus, mitochondria and cytosol). The liver (approximately $5 \mathrm{~g}$ ) was homogenised $1: 10 \mathrm{w} / \mathrm{v}$ in $225 \mathrm{~mm}$ sucrose, $0.3 \mathrm{~mm}$ ethylene glycol-bis (EGTA), $1 \mathrm{~mm}$ 1,4-dithiothreitol and TRIS-HCl (pH 7.4), and it was supplemented with proteases and phosphatase inhibitors at $1500 \boldsymbol{g}$ for $15 \mathrm{~min}$; the supernatant was centrifuged at $10000 \mathbf{g}$ for $20 \mathrm{~min}$ to sediment the mitochondrial fraction. The new supernatant was ultracentrifuged at $100000 \boldsymbol{g}$ for $60 \mathrm{~min}$, yielding the cytosolic fraction (supernatant). The nuclear fraction was prepared from the first pellet using the citric acid method ${ }^{(28)}$.

\section{Metabolite parameters}

The KB and lipid profiles (TAG, cholesterol and lipoproteins) were quantified using a commercial kit (SPINREACT; Lab Center de México S.A. de C.V.), and malonyl-CoA was quantified using a commercial kit (MyBioSource) with an ELISA reader (Bio-Rad Laboratories); all procedures followed the manufacturer's instructions.

\section{Western blot}

Total protein was measured using the Bradford reagent. Equal amounts of proteins $(50 \mu \mathrm{g})$ were mixed with $2 \times$ Laemmli sample buffer (Bio-Rad Laboratories) and incubated at $80^{\circ} \mathrm{C}$ for $1 \mathrm{~min}$. The proteins were separated on a $10 \%$ polyacrylamide gel, electroblotted onto a nitrocellulose membrane, and then incubated overnight with primary antibody Anti-PGC1 $\alpha$ (Abcam) at 1:1000 dilution, Anti-AMPK $\alpha 1$ (2B7) (Abcam) at 1:500 dilution, pAMPK $\alpha$ (Thr172) (40H9) (Cell Signaling Technology) at 1:1000 dilution, Anti-SIRT1 (Abcam) at 1:1000 dilution, Anti-CPT1 $\alpha$, (Cell Signaling Technology) at 1:1000 dilution and Anti-LIPC (Abcam) at 1:1000 dilution. The loading controls were Anti-VDAC1/Porin (Abcam) at 1:1000 dilution, $\beta$-actin (13E5) (Cell Signaling Technology) at 1:2000 dilution for cytosol, and Anti-Lamin B1 (Abcam) at 1:1000 dilution. The membranes were washed and incubated for $2 \mathrm{~h}$ with alkaline phosphatase (AP)-conjugated (goat anti-rabbit; Abcam) secondary antibody at 1:5000 dilution, and the bands were visualised using the AP conjugate substrate kit (Bio-Rad Laboratories) according to the manufacturer's instructions.

\section{Phosphorylated AMP-activated protein kinase $\alpha$ hepatic zonation}

Liver tissue was fixed for $5 \mathrm{~d}$ in $10 \%$ formalin at $4^{\circ} \mathrm{C}$, with regular formalin changes. After fixation, the tissue was embedded in paraffin and sectioned into 7-8 $\mu \mathrm{m}$ slices. Samples were deparaffinised overnight at $60^{\circ} \mathrm{C}$ in a dry-heat oven. Sections were rehydrated in xylol 100\% (10 min), ethanol 100\% (5 min), ethanol 96\% (5 min), ethanol $80 \%$ ( $5 \mathrm{~min}$ ) and deionised water (10 min). Antigen retrieval was performed in saline-sodium citrate Buffer Concentrate $1 \times$ (Sigma-Aldrich) in a water bath at $80^{\circ} \mathrm{C}$ for $25 \mathrm{~min}$, followed by blocking with $1 \%$ non-fat milk for $1 \mathrm{~h}$. The sections were washed $3 \times 5$ min with $0.05 \%$ TRIS-buffered saline plus Tween (TBST) and then incubated overnight at room temperature with the primary antibody against pAMPK $\alpha$ (Thr172) (40H9) at 1:100 dilution. The next day, sections were washed $3 \times 5$ min with $0.05 \%$ TBST and incubated for $2 \mathrm{~h}$ with the secondary antibody Alexa Fluor ${ }^{\circledR} 488$ donkey anti-rabbit IgG (H+L) (Thermo Scientific) at 1:400 dilution. The photos were taken at $40 \times$ magnification. The quantification considered the area and optical density (OD) of the negative and positive signals. For the analysis, ten photos (negative and positive signal) were taken per rat, and ten different hepatocytes were selected from the PV and PP zones. The OD of the nucleus in the PV and PP zones was measured in the first five cell rows. The ratio PP:PV was used to assess the zonation. The criteria were: PP (ratio $>1 \cdot 2$ ), PV (ratio <0.8) and without zonation (ratio 0.8-1.2). The OD was measured using an ImagePro Plus Program version 6.1.

\section{${ }^{14} \mathrm{CO}_{2}$ production}

A quantity of $1 \mathrm{~g}$ of liver was washed in saline solution and homogenised in $2 \mathrm{ml}$ of mannitol buffer: $300 \mathrm{~mm}$ mannitol, $10 \mathrm{~mm}$ HEPES and $1 \mathrm{~mm}$ EDTA ( $\mathrm{pH} 7 \cdot 2$ ). The homogenate was centrifuged at $1800 \boldsymbol{g}$ for $10 \mathrm{~min}$ in a Sorvall RC-5B Plus to obtain the supernatant. A total of $2 \mathrm{mg}$ of homogenate was added to give a volume of incubation solution containing $13 \mathrm{~mm}$ mannitol, $40 \mathrm{~mm}$ $\mathrm{NaCl}, 5 \mathrm{~mm} \mathrm{MgCl}_{2}$, $78 \mathrm{~mm}$ TRIS- $\mathrm{HCl}, 10 \mathrm{~mm} \mathrm{~K}_{2} \mathrm{HPO}_{4}, 2 \mathrm{~mm} \mathrm{KCl}$ and $1 \mathrm{~mm}$ EDTA, followed by a cold solution of $1 \mathrm{~mm}$ L-carnitine, $5 \mathrm{~mm}$ ATP, $1 \mathrm{~mm}$ NAD, $5 \mathrm{~mm}$ ADP, $0.06 \mathrm{~mm}$ FAD and $100 \mu \mathrm{M}$ CoA $(\mathrm{pH} 7 \cdot 2)$ in a $25 \mathrm{ml}$ flask. Finally, $50 \mu \mathrm{l}$ of stock solution of $2 \mathrm{~mm}$ palmitic acid and $\mathrm{U}^{-14} \mathrm{C}$-palmitic acid were added, and the flask was capped with a rubber stopper into the middle of which was inserted a clip that held a $600 \mu$ l Eppendorf Tube containing $100 \mu \mathrm{l}$ of $10 \% \mathrm{KOH}$. The flasks were placed in a water bath at $37^{\circ} \mathrm{C}$ for $90 \mathrm{~min}$. The reaction was terminated by addition of $0.5 \mathrm{ml}$ of $70 \%$ perchloric acid, and the flasks were immediately placed on ice for $60 \mathrm{~min}$. The contents of the Eppendorf Tube were placed in a vial containing $10 \mathrm{ml}$ of Tritosol, and the radioactivity was determined in a cintillation counter; each sample was counted for $2 \mathrm{~min}$. The results are expressed as $\mathrm{pmol} / \mathrm{g}$ per min.

\section{Data analysis}

Power analysis estimated that seventy-two rats (four rats per sampling time) were required to obtain a power of $0 \cdot 080$, when the effect size was moderate (0.030) and a significance level of 0.05 was used $^{(25,26)}$. Data were grouped by experimental condition and time, and are presented as mean values with their standard errors. They were compared by a two-way ANOVA for independent measures with a factor for group (two levels) and a factor for time (eight levels). In order to determine significant time effects for each curve, a one-way ANOVA was performed to detect putative rhythmicity for each group. The one- and two-way ANOVA tests were followed by a Bonferroni post hoc test with the threshold for significance set at $P<0.05$. The Student's $t$ test was applied for feeding condition controls, with the threshold for significance also set at $P<0 \cdot 05$. Statistical analysis was performed with the Graph Pad Prism program (version 5.0 for Windows; GraphPad Software). All graphs were drawn using the Sigmaplot curve-fitting program (Jandel Scientific).

\section{Results}

To address the adaptive response of hepatic lipid metabolism to DRF, parameters corresponding to the FAO process were 
(a)

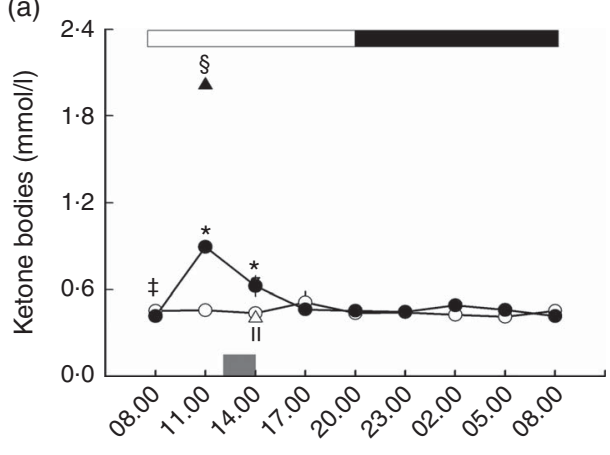

Time (hours)

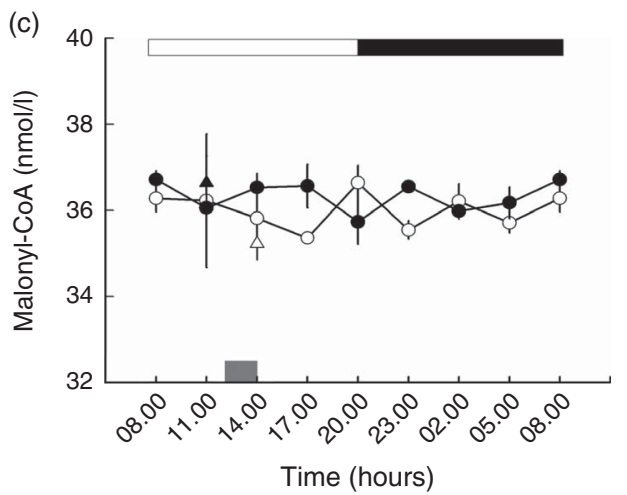

(b)

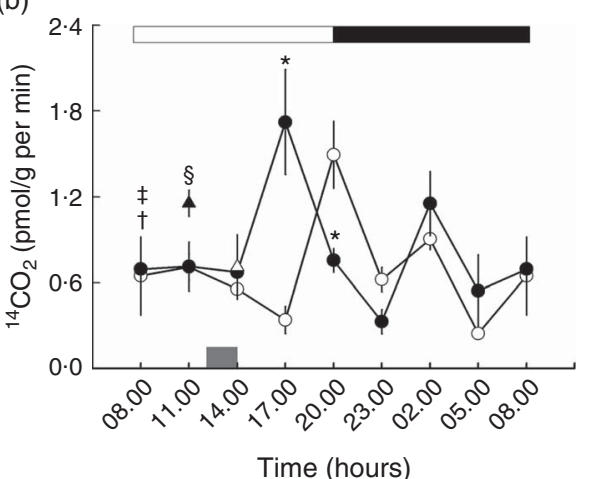

(d)

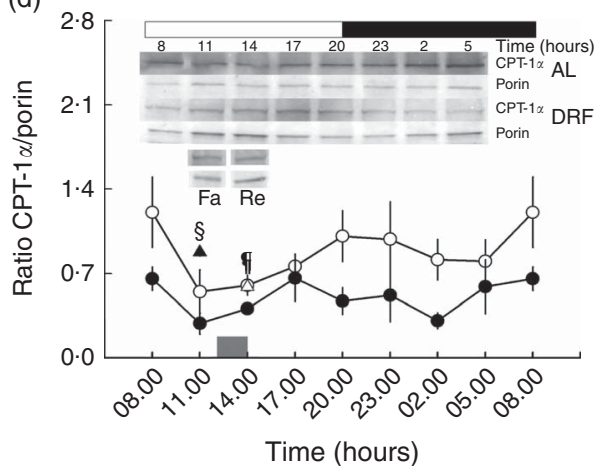

Fig. 1. Effect of restricted feeding on daily variations of the fatty acid oxidation (FAO) pathway. (a) Ketone bodies (serum), (b) ${ }^{14} \mathrm{CO}_{2}$ released by $\mathrm{FAO}$, (c) malonyl-CoA (serum) and (d) carnitine palmitoyl transferase-1a (CPT-1a) protein. Control group fed ad libitum (AL, O), group with daytime restricted feeding (DRF, O), $21 \mathrm{~h}$ fasting group ( $\mathrm{Fa}, \mathbf{\Delta}$ ) and group refed after a 21-h fast (Re, $\Delta$ ). Values are means ( $\mathrm{n} 4 \mathrm{rats}$ ), with their standard errors. $\square$ and $\square, 12 \mathrm{~h}$ light-12h dark periods, respectively; $\square$, time of food access (from 12.00 to 14.00 hours); CPT-1a, carnitine palmitoyltransferase $1 a$. ${ }^{*}$ Time points with a significant difference between the AL and DRF groups (two-way ANOVA, $P<0.05$, Bonferroni post hoc test). $†$ Significant intra-group differences within AL and DRF groups, respectively (one-way ANOVA, $P<0.05$, Bonferroni post hoc test). §§॥ Significant differences of the Fa and Re groups compared with the DRF group (at 11.00 and 14.00 hours), respectively. $\uparrow$ Significant differences between the Fa and Re groups (Student's $t$ test, $P<0.05$ ).

evaluated. Indeed, $\mathrm{KB}$ and $\mathrm{CO}_{2}$ from $\beta$-oxidation are products of mitochondrial FAO in the liver. Fig. 1(a) depicts the 24-h variations in circulating $\mathrm{KB}$. The $\mathrm{AL}$ group showed constant values for serum KB. Certainly, the fasting associated with the resting-sleep period was not enough to promote measurable ketogenesis. In contrast, the DRF group showed a significant increase of approximately $50 \%$ before (at 11.00 hours) and approximately $30 \%$ after (at 14.00 hours) mealtime over the AL group. This elevation represents active ketogenesis in the rats under food restriction. During the light period, KB increased by approximately $23 \%$ in the DRF group. This represents an unexpected metabolic response as the DRF group finishes mealtime at 14.00 hours, and ketogenic activity is usually associated with a fasting condition. The acute feeding control of 21-h fasting (Fa group) showed a $55 \%$ increase in serum $\mathrm{KB}$ over the DRF group (at 11.00 hours). In contrast to the DRF group, $\mathrm{KB}$ in the acute refed rats (Re group) were reduced to the level of the AL group after feeding. Fig. 1(b) shows the daily profile of in vitro ${ }^{14} \mathrm{C}$-palmitic acid oxidation measured as yield of ${ }^{14} \mathrm{CO}_{2}$. Both the groups, $\mathrm{AL}$ and DRF, showed a significant rhythmic profile with a major peak at 20.00 hours (approximately 49\%) and 17.00 hours (approximately 80\%), respectively. DRF rats showed a second, smaller peak at 02.00 hours. In addition, the DRF group showed an average ${ }^{14} \mathrm{CO}_{2}$ production increase of approximately $32 \%$ during the light period in comparison with the $\mathrm{AL}$ group. Meanwhile, the $\mathrm{Fa}$ group showed a significant increase of approximately $50 \%$ in comparison with both the AL and DRF groups (at 11.00 hours). This increase was abolished in the Re group. Complementarily, CPT- $1 \alpha$ (limiting enzyme for mitochondrial uptake of acyl-fatty acids) as well as malonyl-CoA (main metabolite inhibitor of CPT- $1 \alpha$ in response to nutrient uptake) was measured.

Fig. 1(c) and (d) indicate that the levels of malonyl-CoA and CPT- $1 \alpha$ in the liver remained constant in the AL and DRF groups along the day-night cycle. However, the 24-h mean value for CPT- $1 \alpha$ in the DRF rats showed a clear tendency to decrease with respect to the AL group. The acute fasting and refed conditions did not change the malonyl-CoA levels; in contrast, the $\mathrm{Fa}$ group showed a significant increase (approximately $38 \%$ ) in the presence of CPT- $1 \alpha$ in comparison with DRF rats (at 11.00 hours), and the Re group showed an increase of approximately $50 \%$ in CPT- $1 \alpha$ at 14.00 hours. Taken together, these results indicate that in spite of the DRF group having undergone a $22 \mathrm{~h}$ fast, the parameters related to FAO did not show the expected profile of a fasting response.

In order to maintain energetic homoeostasis, the liver uses metabolic sensors such as the AMPK complex. AMPK contains a catalytic subunit $(\alpha)$ and two regulatory subunits $(\beta$ and $\gamma$ ). AMP 
(a)

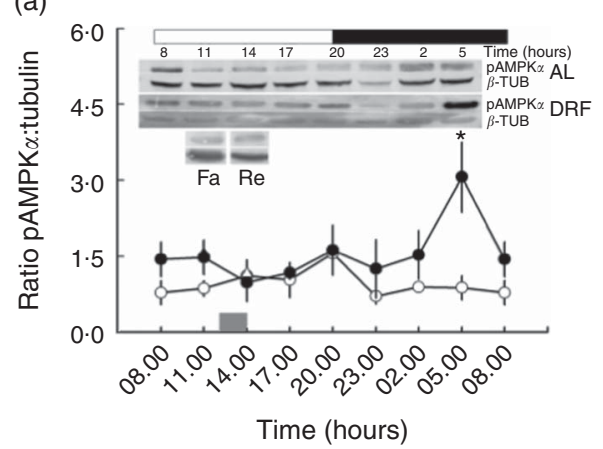

(b)

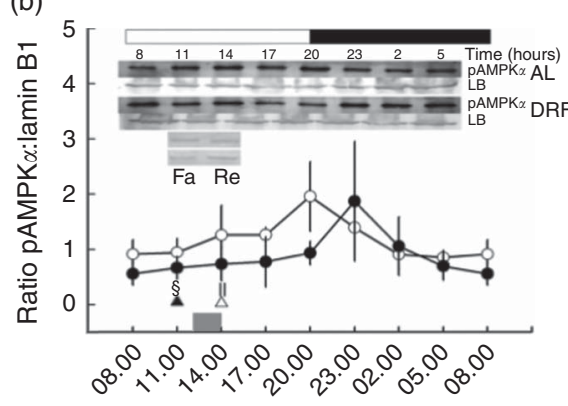

Time (hours)

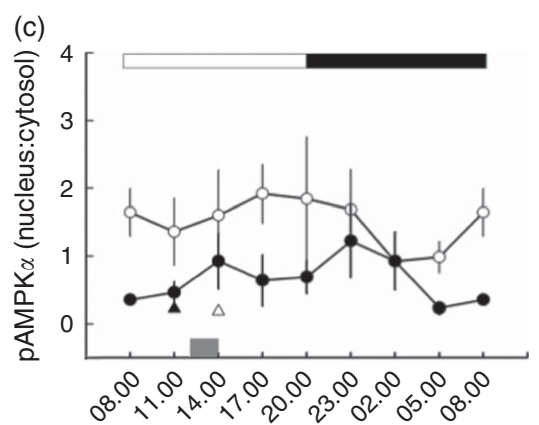

Time (hours)

Fig. 2. Effect of restricted feeding on daily variations of phosphorylated AMP kinase $a$ (pAMPKa) protein in cytosol, nucleus and the ratio of pAMPKa (nucleus:cytosol). (a) AMP-activated protein kinase (AMPKa) (cytosol), (b) AMPKa (nucleus) and (c) AMPKa (nucleus:cytosol). Control group fed ad libitum (AL, O), group with daytime restricted feeding (DRF, ), $21 \mathrm{~h}$ fasting group ( $\mathrm{Fa}, \mathbf{\Delta}$ ) and group refed after a 21 - $\mathrm{h}$ fast (Re, $\triangle$ ). Values are means ( $n 4$ rats), with their standard errors. $\square$ and $\square, 12 \mathrm{~h}$ light-12 $\mathrm{h}$ dark periods, respectively; $\square$, time of food access (from 12.00 to 14.00 hours); $\beta$-TUB, $\beta$-tubulin; LB, laminin B. * Time points with a significant difference between the AL and DRF groups (two-way ANOVA, $P<0.05$, Bonferroni post hoc test). § $\$$ Significant differences of the Fa and Re groups compared with the DRF group (11.00 and 14.00 hours), respectively (Student's $t$ test, $P<0.05$ ).

or ADP can bind to the $\gamma$ subunit under reduced intracellular ATP levels. The full activation of AMPK is accomplished by the serine/threonine kinase LKB1 phosphorylation in $\mathrm{Thr} 172^{(29)}$. Fig. 2(a) depicts the daily profile of the concentration of pAMPK $\alpha$ in cytosol: the AL group showed constant values, whereas the DRF group showed an increase of approximately $50 \%$ at the end of the dark phase (at 05.00 hours). The acute feeding control $\mathrm{Fa}$ and Re groups did not show differences against the DRF group (at 11.00 and 14.00 hours, respectively); however, both groups showed lower values than the DRF group (at 05.00 hours). The $\operatorname{pAMPK} \alpha$ within the nucleus (Fig. 2(b)) showed constant values in the AL and DRF groups, with a subtle increase during the day-night transition in both groups. The Fa and Re groups showed a decrease of 93 and $96 \%$ with respect to the DRF groups (at 11.00 and 14.00 hours, respectively). The $\mathrm{Fa}$ and Re groups showed reduced values in comparison with the AL group average. The nucleus:cytosol ratio of $\mathrm{pAMPK} \alpha$ showed constant daily values in the AL and DRF groups. The DRF group showed a subtle increase of pAMPK $\alpha$ during the day-night transition, with a decrease at the end of the dark phase (at 05.00 hours) that reflected the increase in the cytosolic fraction (at 05.00 hours) (Fig. 2(c)). These data suggest that the DRF group could exhibit pronounced changes in energetic status adaptation throughout the $24 \mathrm{~h}$ cycle compared with the AL group.

Heterogeneous distribution of biochemical pathways through the hepatic acini is known as liver zonation. PP and PV regions exhibit distinct responses to dietary conditions. Liver zonation of the pAMPK $\alpha$ is shown in Fig. 3. In the AL group, this enzyme is present in the PV zone at 11.00 hours, but the zonation is lost at 14.00 and 05.00 hours. In contrast, there was no preferential zonation of pAMPK $\alpha$ in the DRF group at 11.00 hours, but it became PP at 14.00 and 05.00 hours. No zonation was observed in the pAMPK $\alpha$ within the Fa or Re feeding condition controls. The differential compartmentalisation observed in the DRF group responds to an adaptive process distinct to the acute feeding groups.

On the other hand, SIRT1 is another key metabolic sensor of energy status; it regulates glucose and lipid metabolism in the liver, and it promotes gluconeogenesis and $\mathrm{FAO}^{(30)}$. Fig. 4(a) depicts the daily profile of SIRT1. The AL and DRF groups showed constant values of SIRT1 in the nucleus. However, the Fa group showed a marked increase in SIRT1 (approximately 58\%) over the DRF group (at 11.00 hours) and approximately $39 \%$ over the Re group. The acute Re group showed a subtle increase of approximately $15 \%$ over the DRF group (at 14.00 hours). The nuclear:cytosolic ratio of SIRT1 presence was also calculated (Fig. 4(b)) on the basis of previously reported data that used the same experimental DRF protocol $^{(15)}$. This ratio showed a non-rhythmic pattern in the AL group and a clear elevation (approximately 53\%) in the dark period (02.00 and 05.00 hours) in the DRF group. Indeed, this increase was due to a reduction in the cytosolic SIRT1 of DRF rats at those times. The feeding condition controls, the Fa and Re groups (at 11.00 and 14.00 hours), showed values similar to the AL groups, but they showed significant increases of approximately 55 and $35 \%$, respectively, in comparison with the DRF group. Once more, the DRF condition showed a distinct pattern not observed in the acute fasting and refed groups, highlighting a different energetic regulation.

PGC-1 $\alpha$, another molecule responsible for energetic and lipid metabolism homoeostasis was evaluated. Fig. 5(a) shows the daily profile of PGC- $1 \alpha$ in the cytosolic fraction of the AL and DRF groups. The data indicated no differences between the two groups except a tendency for higher values at the light-dark transition (at 20.00 hours). Again, from data previously reported using the same experimental protocol of $\mathrm{DRF}^{(14)}$, the nuclear: cytosolic ratio for the distribution of PGC- $1 \alpha$ was also calculated (Fig. 5(b)). Both the AL and DRF groups showed an increase at 23.00 hours that was statistically significant in the DRF rats, but the main difference was a significant reduction (approximately $68 \%$ ) in the DRF rats throughout the entire day-night cycle. This diminution in the nuclear:cytosolic ratio of PGC- $1 \alpha$ is explained by the striking elevation of this factor in the cytosolic fraction of the DRF rats. The differential distribution of PGC- $1 \alpha$ suggests that the DRF protocol promotes a distinct regulation of energetic status along the $24 \mathrm{~h}$ cycle, accompanied by a reduction of the transcriptional activity associated with PGC- $1 \alpha$.

The 24-h variation of the serum lipid profile is shown in Fig. 6. Total cholesterol levels are depicted in Fig. 6(a): the AL group 
(a)
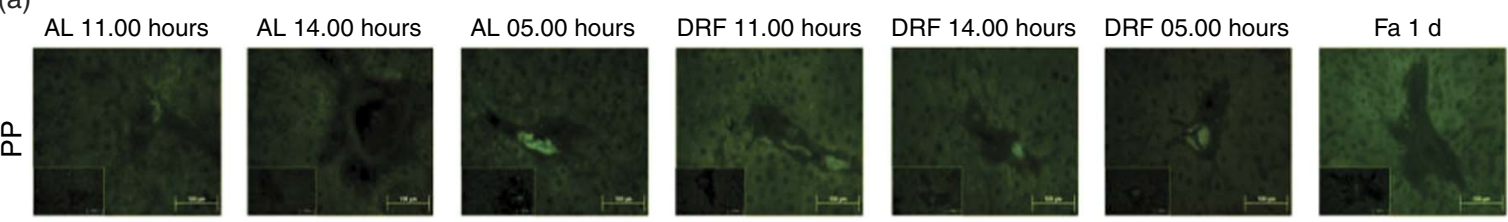

$\operatorname{Re} 1 \mathrm{~d}$
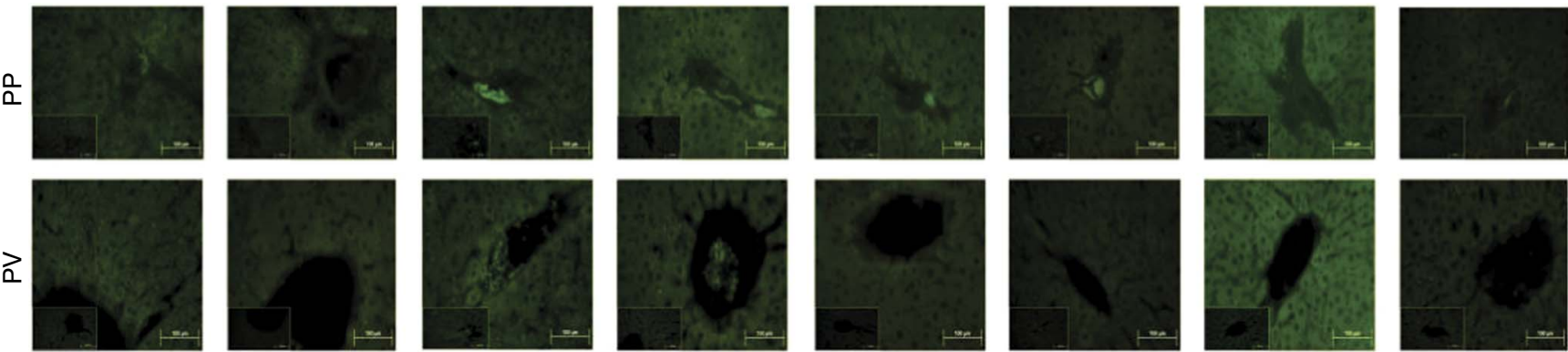

(b)

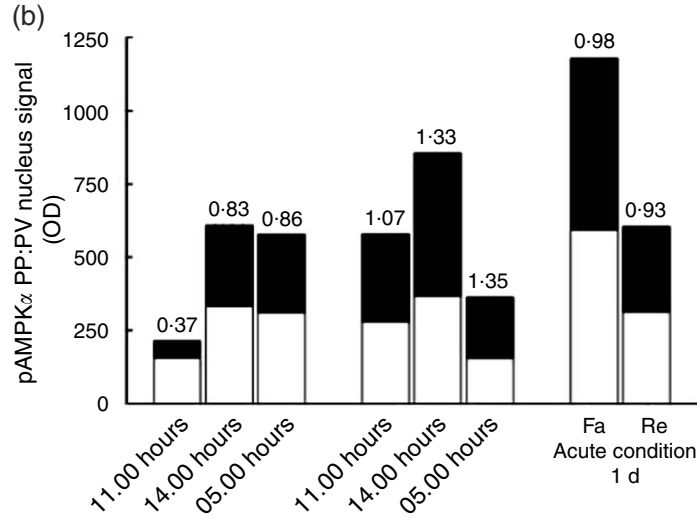

$\mathrm{AL}$

DRF

Fig. 3. Zonal distribution of phosphorylated AMP kinase $a(\mathrm{pAMPKa})$ in the perivenous (PV) and periportal (PP) hepatic nucleus. (a) Immunohistochemical signal for pAMPKa in ad libitum (AL) and daytime restricted feeding (DRF) groups (11.00, 14.00 and 05.00 hours), $21 \mathrm{~h}$ fasting group (Fa) and refed after a 21 -h fast (Re) groups in the portal and central zone. $\square$, Magnifications, 40x scales. Insets included in images of PV and PP hepatocytes are representative of control slices without primary antibody. (b) Histogram showing quantification (optical density (OD)) of the pAMPKa signal in PP ( $\square$ ) and PV $(\square)$ hepatocytes. The ratio between the OD measured in PV and PP hepatocytes is depicted at the top of each column. Hence, distribution can be PP $(>1 \cdot 2), \mathrm{PV}(<0.8)$ or without zonation $(0.8-1 \cdot 2)$. Values are means of at least 200 independent observations of individual hepatocyte nuclei.
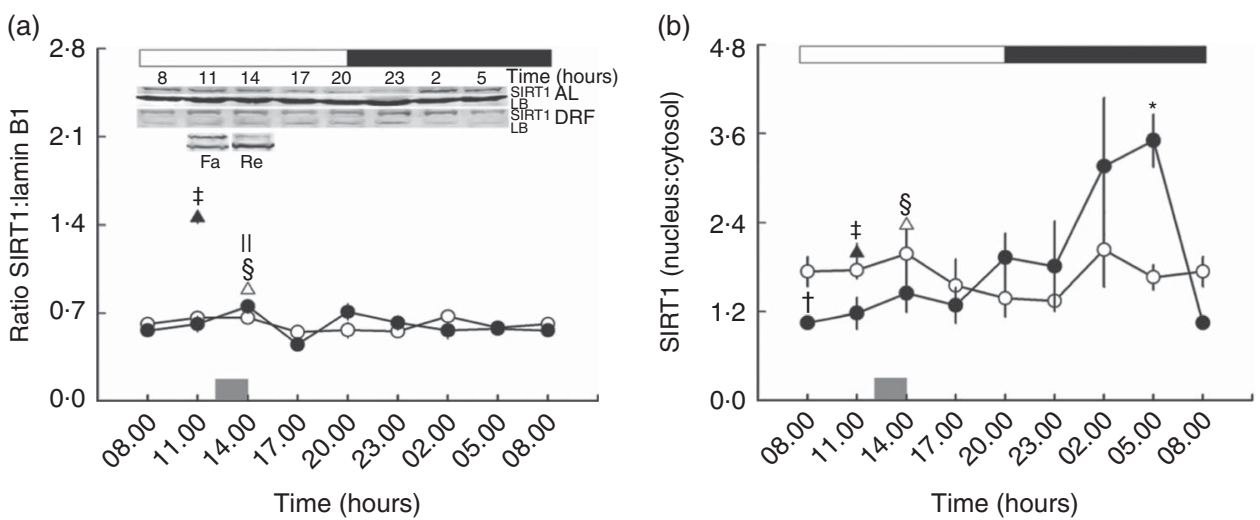

Fig. 4. Effect of restricted feeding on daily variations of silent mating type information regulation homolog 1 (SIRT1) protein in the nucleus and the ratio of SIRT1 (nucleus:cytosol). (a) SIRT1 (nucleus) and (b) SIRT1 (nucleus:cytosol). Control group fed ad libitum (AL, O), group with daytime restricted feeding (DRF, O), $21 \mathrm{~h}$ fasting group ( $\mathrm{Fa}, \triangle$ ) and group refed after a 21 - $\mathrm{h}$ fast (Re, $\square$ ). Values are means ( $\mathrm{H} 4 \mathrm{rats}$ ) with their standard errors. $\square$ and $\square, 12 \mathrm{~h}$ light-12 $\mathrm{h}$ dark periods, respectively; $\square$, time of food access (from 12.00 to 14.00 hours); LB, Laminin B. * Time points with a significant difference between the AL and DRF groups (two-way ANOVA, $P<0.05$, Bonferroni post hoc test). † Significant intra-group differences within DRF groups (one-way ANOVA, $P<0.05$, Bonferroni post hoc test). $\ddagger \S$ Significant differences of the Fa and Re groups compared with the DRF group (11.00 and 14.00 hours), respectively. II Significant differences between the Fa and Re groups (Student's $t$ test, $P<0.05$ )

showed no rhythmicity, whereas the DRF rats exhibited two peaks at 17.00 and 02.00 hours. In addition, the total cholesterol was higher (approximately $20 \%$ ) over the 24-h cycle in the DRF group. This parameter in the Fa and Re groups showed values similar to those of the AL group. In Fig. 6(b), the 24-h profile of serum TAG is shown. The AL and DRF groups showed rhythmic variations; $\mathrm{AL}$ rats displayed peaks at 11.00 and 02.00 hours, whereas the DRF group showed a valley at $11.00 \mathrm{~h}$, just before food access. TAG levels in the $\mathrm{Fa}$ and Re groups showed no differences and their values were similar to the DRF group. In Fig. 6(c), the levels of HDL 
(a)

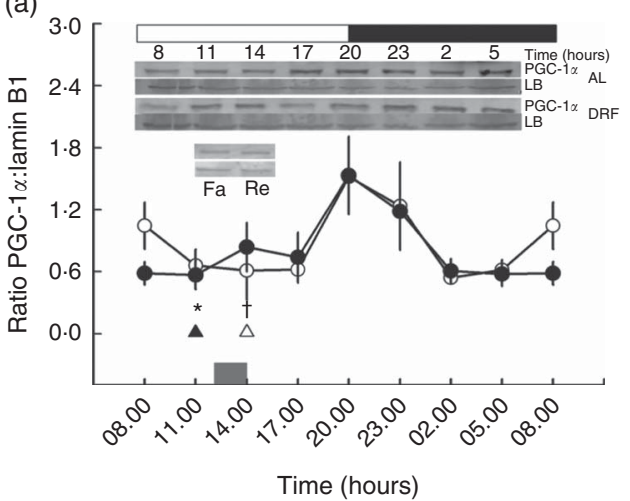

(b)

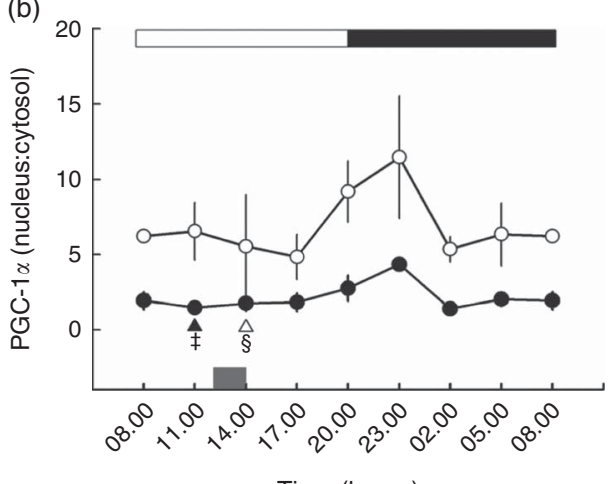

Time (hours)

Fig. 5. Effect of restricted feeding on daily variations of PPARy-1a coactivator (PGC-1a) protein in the nucleus and the distribution of PGC-1a (nucleus:cytosol). (a) PGC-1a (nucleus) and (b) PGC-1a (nucleus:cytosol). Control group fed ad libitum (AL, O), group with daytime restricted feeding (DRF, O), 21h fasting group

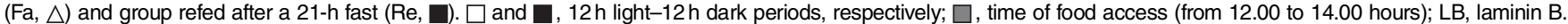
Values are means ( $n 4$ rats) with their standard errors. ${ }^{*}+$ Significant intra-group differences within AL and DRF groups, respectively (one-way ANOVA, $P<0.05$, Bonferroni post hoc test). $\ddagger \S$ Significant differences of the Fa and Re groups compared with the DRF group (11.00 and 14.00 hours), respectively (Student's $t$ test, $P<0.05)$.

(a)

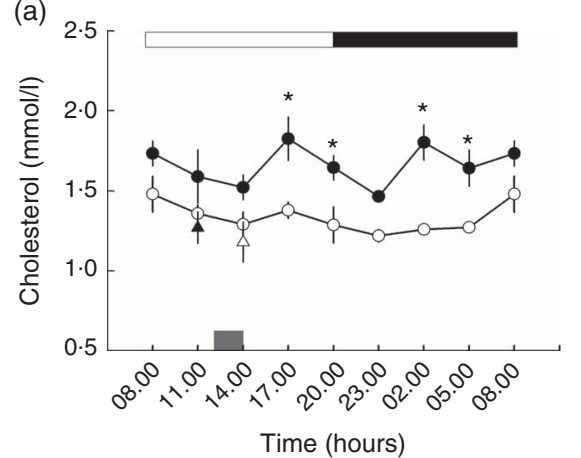

(d)

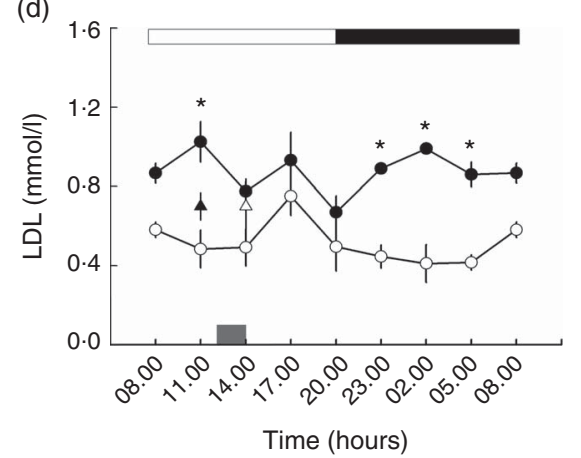

(b)

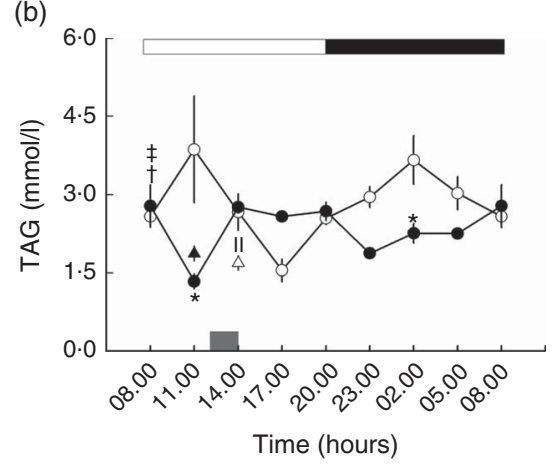

(10)

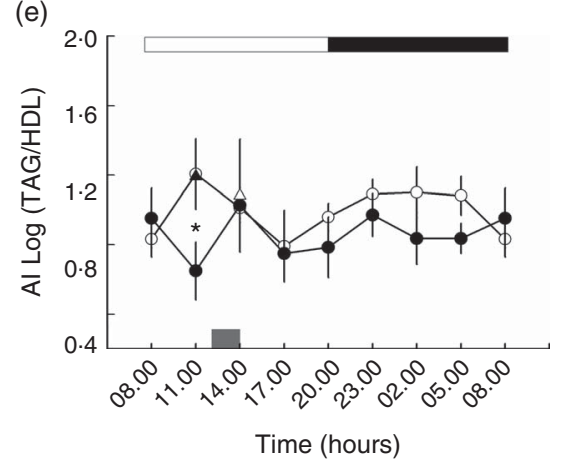

(c)

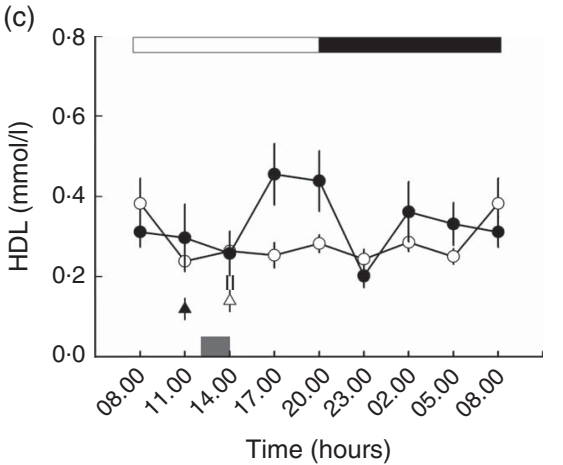

(f)

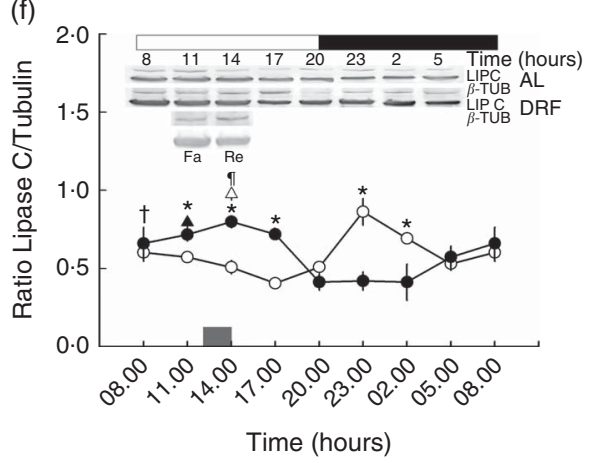

Fig. 6. Effect of restricted feeding on daily variations of the lipid profile in serum and hepatic lipase (LIPC) protein. (a) Cholesterol, (b) TAG, (c) HDL, (d) LDL, (e) atherogenic index (AI) and (f) lipase C. Control group fed ad libitum (AL, O), group with daytime restricted feeding (DRF, O), $21 \mathrm{~h}$ fasting group (Fa, $\mathbf{A}$ ) and group refed after a 21-h fast (Re, $\triangle$ ). Values are means ( $n 4$ rats), with their standard errors. $\square$ and $\square$, 12h light-12h dark periods, respectively; $\square$, time of food access (from 12.00 to 14.00 hours); $\beta$-TUB, $\beta$-tubulin. * Time points with a significant difference between the AL and DRF groups (two-way ANOVA, $P<0.05$, Bonferroni post hoc test). $¥ \ddagger$ Significant intra-group differences within AL and DRF groups, respectively (one-way ANOVA, $P<0.05$, Bonferroni post hoc test). $\|$ Significant difference of the Re group compared with the DRF group (14.00 hours) (Student's $t$ test, $P<0.05)$. I Significant differences between Fa and Re groups (Student's $t$ test, $P<0.05$ ).

are shown. No rhythmicity was detected in AL rats, whereas DRF rats showed higher values at 17.00 and 20.00 hours. Acute fasting and refeeding promoted a significant reduction (approximately $50 \%$ ) in circulating HDL. The 24-h profile of serum LDL (Fig. 6(d)) was similar to the one observed with cholesterol (Fig. 6(a)). The DRF group showed a significant increase (approximately 50\%) in $\mathrm{LDL}$ relative to the $\mathrm{AL}$ group throughout the 24-h cycle. Values of LDL in the $\mathrm{Fa}$ and Re groups were similar to those shown by the DRF rats. Fig. 6(e) shows the index log (TAG/HDL). The 24-h profile of the AL group showed an approximately $24 \%$ increase over DRF rats, with a peak at 11.00 hours. The DRF group showed a valley at 11.00 hours before food access with a tendency towards lower values in the dark period. The Fa group showed a tendency to increase compared with the Re group and DRF rats. Finally, LIPC in the AL group showed a 24-h rhythm with a significant peak during the dark phase of the feeding period 
Table 1. Comparison between groups fed ad libitum (AL) and under daytime restricted feeding (DRF) of 24-h average values*

(Mean values with their standard errors)

\begin{tabular}{|c|c|c|c|c|}
\hline \multirow[b]{3}{*}{ Parameters } & \multicolumn{4}{|c|}{ Groups } \\
\hline & \multicolumn{2}{|c|}{$\mathrm{AL}$} & \multicolumn{2}{|c|}{ DRF } \\
\hline & Mean & SEM & Mean & SEM \\
\hline KB & $2 \cdot 61$ & 0.06 & 3.09† & 0.33 \\
\hline${ }^{14} \mathrm{CO}_{2}$ & 0.69 & 0.14 & 0.82 & 0.03 \\
\hline Malonyl-CoA & $11 \cdot 31$ & 0.04 & 11.41 & 0.03 \\
\hline СРТ-1a & 0.84 & 0.07 & $0.49 \dagger$ & 0.05 \\
\hline SIRT1 (nucleus) & 0.60 & 0.01 & 0.60 & 0.03 \\
\hline SIRT1 (n:c) & 1.68 & 0.09 & $1.92 \dagger$ & 0.33 \\
\hline pAMPKa (cytosol) & 0.98 & 0.09 & 1.57 & 0.23 \\
\hline pAMPKa (nucleus) & $1 \cdot 20$ & 0.14 & 0.93 & 0.15 \\
\hline pAMPKa (n:c) & 1.50 & 0.13 & $0.68 \dagger$ & 0.12 \\
\hline PGC-1a (nucleus) & 0.86 & 0.13 & 0.83 & 0.12 \\
\hline PGC-1a (n:c) & 6.94 & 0.80 & $2 \cdot 19 \dagger$ & 0.34 \\
\hline Cholesterol & 50.91 & $1 \cdot 13$ & $63.89 \dagger$ & 1.75 \\
\hline TAG & $110 \cdot 36$ & $9 \cdot 84$ & 89.49 & $6 \cdot 88$ \\
\hline HDL & 10.61 & 0.63 & $12 \cdot 82$ & 1.17 \\
\hline LDL & 19.69 & 1.52 & $33.85 \dagger$ & 1.56 \\
\hline LIPC & 0.58 & 0.04 & 0.58 & 0.05 \\
\hline Al & 0.65 & 0.05 & $0.50 \dagger$ & 0.04 \\
\hline
\end{tabular}

$\mathrm{KB}$, ketone bodies; CPT-1a, carnitine palmitoyltransferase $1 a$; SIRT1, silent mating type information regulation homolog $1 ; n: c$, ratio of nucleus:cytosol; pAMPKa, phosphorylated AMP-activated protein kinase $a$; LIPC, hepatic lipase; AI, atherogenic index.

* Averages were taken from data in Fig. 1, 2, 4, 5 and 6.

† Mean value was significantly different from that of the $\mathrm{AL}$ group $(P<0.05$; Student's $t$ test); four independent experimental observations.

(23.00 hours). The daily pattern of the DRF group showed a phase shift with a similar increase during the light phase, coincident with the time of food access. Acute feeding control conditions showed an elevation (approximately 20\%) in the refed rats in comparison with the DRF rats (at 14.00 hours) and fasting rats (at 11.00 hours) (Fig. 6(f)). Hence, the DRF condition impacts lipid metabolism and their daily patterns.

Table 1 shows the average parameters of lipid metabolism for the AL and DRF groups. In the AL group, increases in CPT- $1 \alpha$, $\operatorname{pAMPK} \alpha$ (nucleus:cytosol), PGC- $1 \alpha$ (nucleus:cytosol) and atherogenic index (AI) are observed, whereas in the DRF group KB, SIRT1 (nucleus:cytosol), cholesterol and LDL are elevated.

\section{Discussion}

DRF involves a hypoenergetic food intake and a daily synchronisation to food access ${ }^{(2,3)}$. This combination underlies emergent metabolic regulations that influences lipid metabolism in the liver and adipose tissue. The metabolic parameters regulated under the FEO expression showed a rheostatic adaptation in contrast to the conventional acute fasting and refeeding ${ }^{(1,8)}$. This condition was suggestive of an up-regulation of the lipolytic activity in adipose tissue as well as of a temporal regulation of $\beta$-oxidation in the liver ${ }^{(3,9)}$.

\section{Metabolic implications}

Upon the arrival of NEFA, hepatic FAO produces high levels of acetyl-CoA, which is converted into KB within mitochondria ${ }^{(31)}$.
Our results showed an increase in circulating $\mathrm{KB}$ in the DRF group before food access (Fig. 1(a)). KB generation is regulated by the glucagon:insulin ratio under fasting or energy-restricted conditions $^{(32)}$; glucagon increases $\mathrm{KB}$ production, whereas insulin decreases it. The DRF protocol involves high levels of glucagon and reduced circulating insulin before mealtime ${ }^{(7,14)}$. In acute fasting, greater production of KB starts when glycaemia drops several hours after food ingestion ${ }^{(31)}$ (Fig. 1(a)). However, in the DRF rats, serum KB showed a more discreet elevation. This result suggests that after twenty-one cycles of DRF, the fasting response is different, perhaps due to the hyperphagia associated with the slow emptying of the gastric chamber, as well as to the elevated content of hepatic glycogen and the reduction of visceral adipose tissue ${ }^{(3,10)}$.

In the liver, NEFA are taken up and activated to form acylderivatives with COA. Acyl-CoA and their metabolites can act as ligands of the PPAR as well as substrates for peroxisomal and mitochondrial $\mathrm{FAO}^{(33)}$. Complete mitochondrial $\mathrm{FAO}$ produces $\mathrm{CO}_{2}^{(34,35)}$. The AL rats showed the highest levels of ${ }^{14} \mathrm{CO}_{2}$ from FAO during the light-dark transition before mealtime, when the lipolytic activity is expected; in contrast, the DRF group showed two peaks of ${ }^{14} \mathrm{CO}_{2}$ from FAO (at 17.00 and 02.00 hours) (Fig. 1(b)). The production of ${ }^{14} \mathrm{CO}_{2}$ (at 17.00 hours) could be the result of a sudden reduction in insulin levels already reported in the DRF group $^{(7,14)}$. This pattern could be interpreted as an 'out-of-time' fasting response as it is coincident with an elevation of liver PEPCK activity in DRF rats ${ }^{(14)}$. The peak at 02.00 hours is smaller and does not coincide with elevated $\mathrm{KB}$. FAO promotes the production of acetyl-CoA that can enter into the Krebs cycle or be used for ketogenesis. This result could be explained by an enhanced entry of acetyl-CoA into the Krebs cycle instead of $\mathrm{KB}$ production ${ }^{(31,34)}$.

$\operatorname{PPAR} \alpha$ regulates the FAO pathway via CPT- $1 \alpha$. The presence of $\operatorname{PPAR} \alpha$ in the liver showed a peak before food intake in DRF rats; this peak is coincident with the peak of acyl-CoA oxidase, which is the limiting step in the peroxisomal $\mathrm{FAO}^{(9,36)}$. FAO is also controlled by malonyl-CoA, an allosteric inhibitor of CPT- $1 \alpha^{(22)}$. CPT- $1 \alpha$ and malonyl-CoA levels were not significantly different between the AL and DRF groups (Fig. 1(c) and (d)). In contrast, the acute fasting control showed an evident increase in CPT- $1 \alpha$ expression (Fig. 1(d)), indicating the correct sensitivity of our assay. Despite being lower in DRF rats, the CPT- $1 \alpha$ concentration would be enough to activate NEFA and carry out their oxidation (Fig. 1(d)). At the same time, our results showed higher levels of ${ }^{14} \mathrm{CO}_{2}$ production from ${ }^{14} \mathrm{C}$-palmitoyl CoA over a 24 -h period in DRF rats (Table 1 ), which could mean that CPT- $1 \alpha$ could be regulated by covalent modifications or by the mitochondrial membrane microenvironment to favor Krebs cycle ${ }^{(21,34)}$. The physicochemical properties of the membrane and the effects of $\mathrm{pH}$ on malonyl-CoA promote conformational changes in CPT- $1 \alpha$ in response to hormonal and nutritional states that determine the kinetic characteristics of the protein ${ }^{(21,37)}$.

\section{Metabolic regulators (phosphorylated AMP-activated protein kinase, silent mating type information regulation homolog 1 and PPAR $\gamma-1 \alpha$ coactivator)}

The liver shows metabolic plasticity that is necessary for energy homoeostasis $^{(38)}$. Part of this plasticity is the metabolic zonation 
observed in PV and PP hepatocytes; gluconeogenesis, ureagenesis, ketogenesis and $\beta$-oxidation are predominant in the PP zone, whereas glycolysis and lipogenesis are more active in the PV zone. In the fasting state, both mitochondrial FAO and ketogenesis predominate in PP hepatocytes ${ }^{(39)}$. In addition, glucagon promotes the activation of AMPK and elevates FAO during fasting ${ }^{(40)}$. AMPK is an energy sensor that is activated by exercise, starvation and energy restriction; it is located mainly in the PP zone, which is coincident with the presence of glucagon receptors ${ }^{(41)}$. Overall, the changes in liver zonation of pAMPK detected in this project, as well as the changes reported for $\mathrm{PEPCK}^{(14)}$, are indicative of adaptation in cellular plasticity that takes place in the liver during the expression of the FEO.

$\operatorname{pAMPK} \alpha$ was present in the nucleus within the PV zone in the AL rats at 11.00 hours (Fig. 2(b)); its presence in hepatic nuclei showed an enhanced PP zonation in the DRF group (14.00 and 05.00 hours). pAMPK $\alpha$ also leads to inhibition of lipogenesis and glucose production ${ }^{(42)}$. It is known that AMPK $\alpha$ is phosphorylated and activated by LKB1 in response to stress $^{(29)}$. The DRF protocol could involve some stress; for example, the hyperphagia that results in gastric distention after feeding and the increase in NF- $\kappa \mathrm{B}$ within the hepatocyte nuclei after mealtime ${ }^{(2)}$ might cause an activation of AMPK independent of energy and nutritional status. That possibility remains to be tested. The acute fasting group showed a higher presence of pAMPK $\alpha$ compared with the DRF group at 11.00 hours, but without zonation, indicating that DRF rats adapt differently to the processing of nutrients by the liver. The hepatic zonation of pAMPK $\alpha$ in the AL and DRF groups is temporary; this implies distinctive dynamic regulation and metabolic actions of pAMPK $\alpha$ in both groups.

Deacetylase SIRT1 responds to the $\mathrm{NAD}^{+}: \mathrm{NADH}$ ratio when the energy status is low, usually during fasting and energy restriction. Nuclear SIRT1 acts on a variety of transcription factors and coactivators such as forkhead box (FoxO) and PGC- $1 \alpha$, promoting the up-regulation of genes involved in gluconeogenesis and FAO (GPase, PEPCK and CPT-1 $\alpha)^{(20,43,44)}$. The presence of cytosolic SIRT1 ${ }^{(14)}$ showed an increase before and after mealtime in DRF; this event is coincident with the increase of PEPCK activity ${ }^{(14)}$. Despite the fact that nuclear SIRT1 did not show differences between the AL and DRF groups, the nucleus:cytosol ratio of SIRT1 distribution showed a significant increase at 05.00 hours. This result could be explained by the SIRT1 transit into and out of the nucleus, which varies in response to the energy status $\left(\mathrm{NAD}^{+}\right.$availability) ${ }^{(45)}$. The increase of nuclear SIRT1 at the end of the dark phase (Fig. 4(a)) could be associated with the deacetylation of PGC- $1 \alpha$ and FoxO during the activation of gluconeogenesis and $\mathrm{FAO}$, resulting in the increase of circulating KB (Fig. 1(a)) as well as PEPCK activation in DRF rats ${ }^{(14,17)}$.

PGC- $1 \alpha$ regulates the transcriptional activation of $\operatorname{PPAR} \alpha$ as part of a global control of the fasting response ${ }^{(46)}$. In this condition, the expression of PGC- $1 \alpha$ is synergistically induced by glucagon and glucocorticoids ${ }^{(47)}$. The DRF protocol promotes the increase of circulating glucagon and glucocorticoids, especially before the time of food access. Some of the principal effects of glucagon and glucocorticoids are the hydrolysis of TAG in the adipose tissue and the regulation of FAO and gluconeogenesis in the liver ${ }^{(13,48)}$. The effects of glucagon and glucocorticoids could be associated with the increase of cytosolic PGC-1 $\alpha$ (approximately $40 \%$ ) in the 24 -h profile under the DRF condition ${ }^{(14)}$. However, its concentration in nuclear fractions showed no changes, an effect similar to that observed with SIRT1. The AL rats showed a $78 \%$ increase in the nucleus: cytosol ratio of SIRT1 distribution (at 05.00 hours); however, the effect of PGC- $1 \alpha$ on the regulation of the gluconeogenic enzymes, PEPCK and GPGase, as well as on FAO can be observed under the DRF condition (Fig. 1(b) $)^{(14)}$.

\section{Lipoprotein metabolism}

Lipoprotein metabolism responds to fasting and feeding cycles. After food intake, the formation and assembly of VLDL is regulated by Apo B100 availability as well as by the formation of TAG within the liver ${ }^{(23,49)}$. Circulating VLDL are captured and hydrolysed to LDL in extrahepatic tissues. In the fasting state, TAG are hydrolysed in the adipose tissue to release NEFA and glycerol in response to catabolic hormones (e.g. glucagon, adrenaline and glucocorticoids); eventually, NEFA are oxidised in the liver and in skeletal and cardiac muscles ${ }^{(13)}$.

The liver is the major organ that controls LDL transformation; approximately $50 \%$ of LDL molecules are degraded by the liver in a process mediated by Apo B and hepatic LDL receptors ${ }^{(49)}$. Changes in the expression of LDL receptors can modify the levels of circulating $\mathrm{LDL}^{(50)}$ and the cholesterol requirements of different organs. Apo E plays a role in the clearance of remnant lipoproteins by the liver; it is necessary to keep cholesterol levels low in plasma and participates in the uptake system for $\mathrm{LDL}^{(51)}$. Unpublished microarray results from our group indicate a significant decrease of $A p o E$ mRNA (at 08.00 and 14.00 hours) in livers of the DRF group (Table 2). Thus, the potential decrease of Apo E, and the reduction of serum VLDL could be related to the higher levels of LDL (approximately 50\%) and cholesterol (approximately 20\%) observed in the DRF condition. An injection of methylprednisolone in male Wistar rats promotes the increase of LDL in serum ${ }^{(52)}$; data previously reported showed an increase (approximately 20\%) in the 24-h profile of serum corticosteroids in DRF rats ${ }^{(2)}$.

The paraoxonase enzyme that prevents the formation of atherogenic oxidised-LDL ${ }^{(53)}$ showed an increase (Table 2) at 11.00 hours (unpublished microarray results). This suggests that the lipoprotein profile changes in a coordinated response during the DRF protocol/FEO expression. The physiological implications remain to be explored, but it is interesting that the atherogenic index was lower in the DRF rats (Fig. 6(f)).

Earlier studies showed a reduction of circulating and hepatic TAG before food access in the DRF group ${ }^{(8,10)}$. The LIPC hydrolyses TAG and phospholipids, promoting the uptake of HDL by liver, and converts intermediate-density lipoprotein into $\mathrm{LDL}^{(54)}$. In the DRF group, the increase of LIPC before mealtime coincides with a reduction of TAG in liver ${ }^{(10)}$. Another enzyme, lipoprotein lipase (LPL), hydrolyses circulating chylomicrons and VLDL ${ }^{(55)}$. Processing of VLDL by LPL results in the formation and cholesterol and apoE ${ }^{(56)}$. Unpublished microarray results showed an increase of hepatic $L P L$ (Table 2), before food intake that could be associated with the reduction of serum TAG and VLDL. HDL 
Table 2. Analysis of the expression of genes involved in lipid management in liver of rats with food restriction by micro-arrangements

\begin{tabular}{|c|c|c|c|}
\hline \multirow[b]{2}{*}{ Genes implicated in lipid management } & \multicolumn{3}{|c|}{ Time (hours) } \\
\hline & 08.00 & 11.00 & 14.00 \\
\hline Apo A-I (HDL) & & 44.8 & \\
\hline Apo A-II (HDL) & & 43.3 & \\
\hline Apo B (CM, VLDL, LDL) & & 46.6 & \\
\hline Apo $C-I$ ( $C M, V L D L, L D L)$ & & 446 & \\
\hline Apo C-III (CM, VLDL, LDL) & & 44.4 & \\
\hline Apo $D$ & & & $\downarrow 2 \cdot 2$ \\
\hline Apo E (LDL receptor) & $\downarrow 3.8$ & & $\downarrow 3 \cdot 2$ \\
\hline$L P L$ & & 42.7 & 42.8 \\
\hline Paraoxonase 1 & & 43.6 & \\
\hline
\end{tabular}

$\mathrm{CM}$, chylomicrons; LPL, lipoprotein lipase; $\mathbf{4}$, increase and $\downarrow$, decrease in the gene expression at 08.00 hours (before food anticipatory behaviour), 11.00 hours (food anticipatory behaviour) and 14.00 hours (after food intake) in the daytime restricted feeding group compared with the ad libitum group; six independent experimental observations.

did not show significant differences but the daily profile was modified by the DRF condition.

Unpublished microarray results from our group indicate a significant decrease in ApO E mRNA (at 08.00 and 14.00 hours) in livers of the DRF group (Table 2). Thus, the potential decrease of Apo E, and the reduction of serum VLDL, could be related to higher levels of LDL (approximately 50\%) and cholesterol (approximately 20\%) observed in the DRF condition. An injection of methylprednisolone in male Wistar rats promotes the increase of LDL in the serum ${ }^{(51)}$; data previously reported showed an increase (approximately 20\%) in the 24-h profile of serum corticosteroids in DRF rats ${ }^{(2)}$.

An atherogenic profile is an important risk factor for coronary diseases $^{(57,58)}$. However, changes in serum lipoproteins cannot always be associated with dyslipidaemias; for example, pregnant women show increased serum lipid content but a low cardiac risk factor ${ }^{(59)}$; the $\mathrm{AI}$ is higher in postmenopausal women ${ }^{(60)}$; the lipid profile is atherogenic during pregnancy and lactation in Sprague-Dawley rats ${ }^{(61)}$; high-sucrose and high-fat diets increase TAG in the serum and liver, as well as circulating cholesterol and $\mathrm{LDL}^{(62)}$. According to our results, the DRF group showed a decrease in AI in spite of high LDL levels; this can be explained by the low TAG concentrations in the DRF group (Table 2).

\section{Circadian aspects}

The feeding and fasting states are regulated by the SCN in response to day-night cycles. DRF involves daily cycles that consist of $22 \mathrm{~h}$ of fasting and $2 \mathrm{~h}$ of mealtime for 3 weeks. The circadian clock is regulated through feedback loops; in the positive loop, the proteins circadian locomotor output cycles kaput (CLOCK) and brain and muscle Arnt-like protein 1 (BMAL1) heterodimerise and initiate transcription of the target genes period (Per) and cryptochrome (Cry). In the negative loop, PER and CRY heterodimerise and translocate to the nucleus to repress their own transcription ${ }^{(63)}$

Nutritional sensors can act as modulators of the circadian molecular clock: $\mathrm{NAD}^{+}$inhibits CLOCK-BMAL1 heterodimer activity and indirectly regulates the expression of genes (Bmal1, Per2 and Cry) by binding to deacetylase SIRT1 and promoting the degradation of PER2 ${ }^{(64,65)}$. In addition, CLOCK-BMAL1 regulates the expression of nicotinamide phosphoribosyltransferase (NAMPT), the enzyme that catalyses the rate-limiting step in $\mathrm{NAD}^{+}$synthesis ${ }^{(66)}$. PGC- $1 \alpha$ also acts as an intermediary between metabolism and the circadian clock; down-regulation of PGC- $1 \alpha$ iRNA in the liver disrupts rhythmic transcription of BMAL1, CRY1 and CRY2, and modifies the daily PEPCK cycle ${ }^{(67)}$. DRF promotes a phase shift in BMAL1 and PER1 ${ }^{(2,3)}$, an oxidised redox state in the mitochondria and cytosol $^{(7)}$, higher levels of SIRT1 in hepatic cytosol before mealtime, and an elevated presence of cytosolic PGC- $1 \alpha$ in the $24-\mathrm{h}$ profile ${ }^{(14)}$. All these changes are associated with an enhanced 'fasting' response in DRF rats at times before food access ${ }^{(2,3,7,14)}$. In our results, the nucleus:cytosol ratio of SIRT1 exhibits a peak at the end of the dark phase (Fig. 4(b)); this increase could be associated with chromatin remodellingmediated clock proteins for gluconeogenesis circadian control $^{(14,65)}$, and in the FAO-mediated increase of PGC- $1 \alpha$ in the DRF rats; moreover, the 24-h average of the nucleus:cytosol ratio of PGC- $1 \alpha$ is also modified (Fig. 5(b)). AMPK acts in the bidirectional regulation between metabolism and clockwork through the phosphorylation and degradation of CRY1 and the activation of $\mathrm{NAMPT}^{(68)}$. It has been observed that energy restriction and exercise promote the increase of SIRT1 and AMPK, both being key parts of the PGC- $1 \alpha$-activating process that promotes catabolic metabolism and mitochondrial biogenesis in many tissues by regulating the expression of BMAL1 ${ }^{(69)}$. Under the DRF protocol, the levels of AMP and ATP decreased and increased, respectively, before food access (at 11.00 hours), which could explain the highest levels of pAMPK $\alpha$ (at 05.00 hours) in the cytosolic fraction (Fig. 2(a) $)^{(7)}$.

DRF is characterised by the presence of food anticipatory behaviour (FAB) before mealtime, when the metabolic fasting response is more active in the liver ${ }^{(2,7-9,14)}$. The metabolic products of lipid catabolism, KB (Fig. 1(a)) and NEFA ${ }^{(8,9)}$, showed higher levels during FAB. It was recently demonstrated that $\mathrm{KB}$ are necessary for $\mathrm{FAB}$, and that the liver-specific deletion of PER2 in mice abolished FAB, but the viral overexpression of PER2 restored it by regulating the production of $\mathrm{KB}$ to induce $\mathrm{FAB}$ in restricted feeding ${ }^{(70)}$.

In conclusion, the results provide evidence that DRF leads to differential regulation in the handling of lipids. The bidirectional relation between clockwork and metabolism, which is associated with FEO, highlights the relevance of lipid metabolism (FAO) and its regulatory mechanisms, as well as the lipid profile (cholesterol and LDL) in the liver and extrahepatic tissues under FEO. Further studies are needed to understand the physiological consequences of lipid metabolism in the rheostatic adaptations involved in restricted feeding schedules and the expression of FEO.

\section{Acknowledgements}

The authors are grateful to Dorothy Pless and Jessica M. González-Norris for their assistance with the English version of this manuscript, Leonor Casanova and Lourdes Lara for their academic support, and PhD Olivia Vázquez-Martínez for technical assistance.

This work was supported by Dirección General de Asuntos del Personal Académico, Programa de Apoyo a Proyectos de 
Investigación e Innovación Tecnológica (IN200815) to M. D.-M. J. B. R.-Z. is a PhD student from the Programa de Doctorado en Ciencias Biomédicas, Universidad Nacional Autónoma de México and received fellowship 215711 from CONACYT.

J. B. R.-Z. performed experiments; J. B. R.-Z., R. H.-M. and G. A. B.-R. analysed data; J. B. R.-Z. and M. D.-M. wrote the paper; C. M.-A., M. P.-M., and M. O.-M. performed and interpreted experiments; R. H.-M. and G. A. B.-R. were technical advisers; M. D.-M. designed the study.

The authors declare that there are no conflicts of interest.

\section{References}

1. Aguilar-Roblero R \& Díaz-Muñoz M (2010) Chronostatic adaptations in liver to restricted feeding: the FEO as an emergent oscillator. Sleep Biol Rhythms 8, 9-17.

2. Luna-Moreno D, Aguilar-Roblero R \& Díaz-Muñoz M (2009) Restricted feeding entrains rhythms of inflammation related factors without promoting an acute-phase response. Chronobiol Int 26, 1409-1429.

3. Arellanes-Licea EC, Báez-Ruiz A, Carranza ME, et al. (2014) Daily patterns and adaptation of the ghrelin, growth hormone and insulin-like growth factor-1 system under daytime food synchronization in rats. J Neuroendocrinol 26, 282-295.

4. Damiola F, Le Minh N, Preitner N, et al. (2000) Restricted feeding uncouples circadian oscillators in peripheral tissues from the central pacemaker in the suprachiasmatic nucleus. Genes Dev 14, 2950-2961.

5. Mistlberger RE (1994) Circadian food-anticipatory activity: formal models and physiological mechanisms. Neurosci Biobehav Rev 18, 171-195.

6. Mistlberger RE (2009) Food anticipatory circadian rhythms: concepts and methods. Eur J Neurosci 30, 1718-1729.

7. Díaz-Muñoz M, Vázquez-Martínez $\mathrm{O}$, Aguilar-Roblero R, et al. (2000) Anticipatory changes in liver metabolism and entrainment of insulin, glucagon, and corticosterone in food-restricted rats. Am J Physiol Regul Integr Comp Physiol 279, R2048-R2056.

8. Escobar C, Díaz-Muñoz M, Encinas F, et al. (1998) Persistence of metabolic rhythmicity during fasting and its entrainment by restricted feeding schedules in rats. Am J Physiol 274, R1309-R1316

9. Rivera-Zavala JB, Báez-Ruiz A \& Díaz-Muñoz M (2011) Changes in the $24 \mathrm{~h}$ rhythmicity of liver PPARs and peroxisomal markers when feeding is restricted to two daytime hours. PPAR Res, (Epublication ahead of print version 5 April 2011).

10. Díaz-Muñoz M, Vázquez-Martínez O, Báez-Ruiz A, et al. (2010) Daytime food restriction alters liver glycogen, triacylglycerols, and cell size. A histochemical, morphometric, and ultrastructural study. Comp Hepatol 23, 9.

11. Báez-Ruiz A, Escobar C, Aguilar-Roblero R, et al. (2005) Metabolic adaptations of liver mitochondria during restricted feeding schedules. Am J Physiol Gastrointest Liver Physiol 289, G1015-G1023.

12. Jungermann K (1988) Metabolic zonation of liver parenchyma. Semin Liver Dis 8, 329-341.

13. Rui L (2014) Energy Metabolism in the liver. Compr Physiol 4, 177-197.

14. Pérez-Mendoza M, Rivera-Zavala JB \& Díaz-Muñoz M (2014) Daytime restricted feeding modifies the daily variations of liver gluconeogenesis: adaptations in biochemical and endocrine regulators. Chronobiol Int 3, 815-828.

15. Luna-Moreno D, García-Ayala B \& Díaz-Muñoz M (2012) Daytime restricted feeding modifies $24 \mathrm{~h}$ rhythmicity and subcellular distribution of liver glucocorticoid receptor and the urea cycle in rat liver. Br J Nutr 14, 2002-2013.

16. Hardie G (2007) AMP-activated/SNF1 protein kinases: conserved guardians of cellular energy. Nat Rev Mol Cell Biol 8, 774-785.

17. Rodgers JT, Lerin C, Haas W, et al. (2005) Nutrient control of glucose homeostasis through a complex of PGC-1 alpha and SIRT1. Nature 434, 113-118.

18. Murwarid M, Assifi GS, Constant S, et al. (2005) AMP-activated protein kinase and coordination of hepatic fatty acid metabolism of starved/carbohydrate-refed rats. Am J Physiol Endocrinol Metab 289, E794-E800.

19. Ruderman NB, Park H, Kaushik VK, et al. (2003) AMPK as a metabolic switch in rat muscle, liver and adipose tissue after exercise. Acta Physiol Scand 178, 435-442.

20. Hayashida S, Arimoto A, Kuramoto Y, et al. (2010) Fasting promotes the expression of SIRT1, and $\mathrm{NAD}^{+}$- dependent proteins deacetylase, via activation of PPAR $\alpha$ in mice. Mol Cell Biochem 339, 285-292.

21. Zammit VA (2008) Carnitine palmitoyltransferase 1: central to cell function. IUBMB Life 60, 347-354.

22. McGarry JD, Mannaerts GP \& Foster DW (1977) A possible role for malonyl-CoA in the regulation of hepatic fatty acid oxidation and ketogenesis. J Clin Invest 60, 265-270.

23. Beisiegel U (1998) Lipoprotein metabolism. Eur Heart J 19, A20-A23.

24. Chatterjee C \& Sparks DL (2011) Hepatic lipase, high density lipoproteins and hypertriglyceridemia. Am J Pathol 178, 1429-1433.

25. Cohen J (1977) Statistical Power Analysis for the Behavioral Sciences. New York: Academic Press.

26. Poland RE, Rubin RT \& Morton EW Jr (1980) Circadian patterns of rat anterior pituitary and target gland hormones in serum: determination of the appropriate sample size by statistical power analysis. Psychoneuroendocrinology 5, 209-224.

27. Portaluppi F, Touitou Y \& Smolensky MH (2008) Ethical and methodological standards for laboratory and medical biological rhythm research. Chronobiol Int $\mathbf{2 5}$, 999-1016.

28. Aguilar-Delfín I, López-Barrera F \& Hernández-Muñoz R (1996) Selective enhancement of lipid peroxidation in plasma membrane in two experimental models of liver regeneration: partial hepatectomy and acute CC14 administration. Hepato$\log y$ 24, 657-662.

29. Hardie DG \& Alessi DR (2013) LKB1 and AMPK and the cancer-metabolism link-ten years after. BMC Biol 11, 36.

30. Tanner KG, Landry J, Sternglanz R, et al. (2000) Silent information regulator 2 family of NAD-dependent histone/protein deacetylases generates a unique product, 1-O-acetyl-ADP-ribose. Proc Natl Acad Sci U S A 97, 14178-14182.

31. Balasse EO \& Féry F (1989) Ketone body production and disposal: effects of fasting, diabetes, and exercise. Diabetes Metab Rev 5, 247-270.

32. Reed WD, Baab PJ, Hawkins RL, et al. (1984) The effects of insulin and glucagon on ketone body turnover. Biochem J $\mathbf{1 5}$, 439-444.

33. Yu S, Rao S \& Reddy JK (2003) Peroxisome proliferatoractivated receptors, fatty acid oxidation, steatohepatitis and hepatocarcinogenesis. Curr Mol Med 3, 561-572.

34. Lodish H, Berk A \& Zipursky SL (2000) Molecular Cell Biology, 4th ed., pp. 307-313. New York, NY: W. H. Freeman.

35. Ontko JA (1972) Metabolism of free fatty acids in isolated liver cells. J Biol Chem 247, 1788-1800.

36. Reddy JK \& Hashimoto T (2001) Peroxisomal beta-oxidation and peroxisome proliferator-activated receptor alpha: an adaptive metabolic system. Annu Rev Nutr 1, 193-230. 
37. Stephens TW, Cook GA \& Harris RA (1983) Effect of pH on malonyl-CoA inhibition of carnitine palmitoyltransferase I. Biochem J 212, 521-524.

38. Owen OE, Reichard GA Jr, Patel MS, et al. (1979) Energy metabolism in feasting and fasting. Adv Exp Med Biol 111 , 169-188.

39. Guzman M, Bijleveld C \& Geelen MJ (1995) Flexibility of zonation of fatty acid oxidation in rat liver. Biochem $J$ 31, 853-860.

40. Heimberg M, Weinstein I \& Kohout M (1969) The effects of glucagon, dibutyryl cyclic adenosine 3', 5'-monophosphate, and concentration of free fatty acid on hepatic lipid metabolism. J Biol Chem 244, 5131-5139.

41. Witters LA, Gao G, Kemp BE, et al. (1994) Hepatic 5'-AMPactivated protein kinase: zonal distribution and relationship to acetyl-coA carboxylase activity in varying nutritional states. Arch Biochem Biophys 308, 413-419.

42. Viollet B, Foretz M \& Guigas B (2006) Activation of AMP-activated protein kinase in the liver: a new strategy for the management of metabolic hepatic disorders. J Physiol 574, 41-53.

43. Kwon HS \& Ott M (2008) The ups and downs of SIRT1. Trends Biochem Sci 33, 517-525.

44. Rodgers JT \& Puigserver P (2007) Fasting-dependent glucose and lipid metabolic response through hepatic sirtuin 1. Proc Natl Acad Sci U S A 104, 12861-12866.

45. Tanno M, Sakamoto J \& Miura T (2007) Nucleocytoplasmic shuttling of the NAD+-dependent histone deacetylase SIRT1. J Biol Chem 2, 6823-6832.

46. Vega RB, Huss JM \& Kelly DP (2000) The coactivator PGC-1 cooperates with peroxisome proliferator-activated receptor alpha in transcriptional control of nuclear genes encoding mitochondrial fatty acid oxidation enzymes. Mol Cell Biol 20, 1868-1876.

47. Yoon JC, Puigserver P, Chen G, et al. (2001) Control of hepatic gluconeogenesis through the transcriptional coactivator PGC-1. Nature 413, 131-138.

48. Ahmadian M, Duncan RE, Jaworski K, et al. (2007) Triacylglycerol metabolism in adipose tissue. Future Lipidol 2, 229-237.

49. Fisher EA \& Ginsberg HN (2002) Complexity in the secretory pathway: the assembly and secretion of apolipoprotein B-containing lipoproteins. J Biol Chem 277, 17377-17380.

50. Goldstein JL, Basu SK, Brown MS, et al. (1983) Receptor-mediated endocytosis of low-density lipoprotein in cultured cells. Methods Enzymol 98, 241-260.

51. Kuipers F, Jong MC, Lin Y, et al. (1997) Impaired secretion of very low density lipoprotein-triglycerides by apolipoprotein E-deficient mouse hepatocytes. J Clin Invest 100, 2915-2922.

52. Hazra A, Pyszczynski NA, DuBois DC, et al. (2008) Modeling of corticosteroid effects on hepatic low-density lipoprotein receptors and plasma lipid dynamics in rats. Pharm Res 25, 769-780.

53. Cao H, Girard-Globa A, Berthezene F, et al. (1999) Paraoxonase protection of LDL against peroxidation is independent of its esterase activity towards paraoxon and unaffected by $\mathrm{Q} \rightarrow \mathrm{R}$ genetic polymorphism. J Lipid Res 40, 133-139.
54. Landin B, Nilsson A, Twu JS, et al. (1984) A role for hepatic lipase in chylomicron and high density lipoprotein phospholipid metabolism. J Lipid Res 25, 559-563.

55. Murdoch SJ \& Breckenridge WC (1995) Influence of lipoprotein lipase and hepatic lipase on the transformation of VLDL and HDL during lipolysis of VLDL. Atherosclerosis 118, 193-212.

56. Mead JR, Irvine SA \& Ramji DP (2002) Lipoprotein lipase: structure, function, regulation, and role in disease. $J \mathrm{Mol}$ Med 80, 753-769.

57. Dobiásová M \& Frohlich J (2001) The plasma parameter log (TG/HDL-C) as an atherogenic index: correlation with lipoprotein particle size and esterification rate in apoBlipoprotein-depleted plasma (FER(HDL)). Clin Biochem 34, 583-588.

58. Mertz DP (1980) "Atherosclerosis-index" (LDL/HDL): risk indicator in lipid metabolism disorders. Med Klin 75, 159-161.

59. Neboh EE, Emeh JK, Aniebue UU, et al. (2012) Relationship between lipid and lipoprotein metabolism in trimesters of pregnancy in Nigerian women: is pregnancy a risk factor? J Nat Sci Biol Med 3, 32-37.

60. Nwagha UI, Ikekpeazu EJ, Ejezie FE, et al. (2010) Atherogenic index of plasma as useful predictor of cardiovascular risk among postmenopausal women in Enugu, Nigeria. Afr Health Sci 10, 248-252.

61. Smith JL, Lear SR, Forte TM, et al. (1998) Effect of pregnancy and lactation on lipoprotein and cholesterol metabolism in the rat. J Lipid Res 39, 2237-2249.

62. Ryu MH \& Cha YS (2003) The effects of a high-fat or highsucrose diet on serum lipid profiles, hepatic acyl-CoA synthetase, carnitine palmitoyltransferase-I, and the acetyl-CoA carboxylase mRNA levels in rats. J Biochem Mol Biol 36, 312-318.

63. Ko CH \& Takahashi JS (2006) Molecular components of the mammalian circadian clock. Hum Mol Genet 15, R271-R277.

64. Asher G, Gatfield D \& Stratmann M (2008) SIRT1 regulates circadian clock gene expression through PER2 deacetylation. Cell 134, 317-328.

65. Nakahata Y, Sahar S, Astarita G, et al. (2009) Circadian control of the $\mathrm{NAD}^{+}$salvage pathway by CLOCK-SIRT1. Science $\mathbf{3 2 4}$, 654-657.

66. Nakahata Y, Kaluzova M, Grimaldi B, et al. (2008) The NAD +-dependent deacetylase SIRT1 modulates CLOCKmediated chromatin remodeling and circadian control. Cell 134, 329-340.

67. Liu Q, Dinu I, Adewale AJ, et al. (2007) Comparative evaluation of gene-set analysis methods. BMC Bioinformatics $\mathbf{8}, 431$.

68. Lamia KA, Sachdeva UM, DiTacchio L, et al. (2009) AMPK regulates the circadian clock by cryptochrome phosphorylation and degradation. Science 326, 437-440.

69. Sahar S \& Sassone-Corsi P (2011) Regulation of metabolism: the circadian clock dictates the time. Trends Endocrinol Metab 23, $1-8$.

70. Chavan R, Feillet C \& Costa SS (2016) Liver-derived ketone bodies are necessary for food anticipation. Nat Commun $\mathbf{3}, 7$. 